\title{
DESAFíos Para la SALUd de las VACAS DURANTE EL PERIODO DE TRANSICIÓN. USO DE MONENSINA
}

\author{
Monensin in transition dairy cows
}

\author{
Alcázar, E.; Martínez, S.; Madrid, J.; Larrosa, P.; Hernández, F. \\ Departamento de Producción Animal. Facultad de Veterinaria. Campus de Espinardo, Universidad \\ de Murcia, 30100, Murcia. España.
}

* Autor para correspondencia: Alcázar Triviño, Estefanía. Tel: 630455982. Email: estefania. alcazar@elbarranquillo.es

Historial del artículo:

Recibido: 14 de mayo, 2018

Aceptado: 11 de noviembre, 2018

\section{RESUMEN}

El objetivo de este trabajo fue realizar una revisión de los efectos derivados del uso de monensina como alternativa para afrontar con mayor éxito el periodo de transición en vacas lecheras. En las vacas de leche, la transición de la preñez al inicio de la lactación es un periodo crítico que se caracteriza por presentar una mayor incidencia de enfermedades. En este periodo, el riesgo de que se presenten trastornos metabólicos y enfermedades está relacionado con el balance energético negativo en el que se encuentra la vaca, hecho que conlleva una movilización de nutrientes desde las reservas tisulares. Una de las posibles soluciones para mejorar el balance de nutrientes durante el período de transición consiste en administrar ionóforos como la monensina que actúan modificando la población microbiana del rumen. Los efectos beneficiosos de la monensina se han asociado a la producción de precursores gluconeogénicos a nivel ruminal, lo que originaría mayor disponibilidad de glucosa y la consiguiente mejora del estatus energético. Con el uso de monensina, a nivel sanguíneo se ha observado un descenso de $\beta$-hidroxibutirato, y ácidos grasos no esterificados. También han sido atribuidos efectos beneficiosos sobre la funcionalidad hepática, la absorción de ciertos minerales, la función inmune y la excreción de metano. 
En definitiva, el efecto positivo de la MON sobre el balance energético y la utilización de nutrientes reduciría la movilización de reservas tisulares, minimizando el riesgo de desórdenes metabólicos, mejorando la salud y por tanto el rendimiento de la vaca lechera.

Palabras clave: ionóforos, metabolismo ruminal, vacas de leche.

\section{ABSTRACT}

The aim of this research was to carry out a review of the use of monensin in dairy cows as a choice to approach more successfully the transition period. In dairy cows, the transition for the pregnancy to the beginning of lactation is a critical period which is characterized by display a higher incidence of diseases. The risk of metabolic disorders and other diseases during this time is allied to the state of negative energy balance in which the cow is, fact that involves a mobilization of nutrients from the tissue reserves. One of the possible solutions to improve the nutrient balance during the transition period is based on dispense ionophores such as monensin who modify the microbial population of the rumen. The beneficial effects of monensin have been associated with the production of gluconeogenic precursors at the ruminal level which would lead to greater availability of glucose and the consequent improvement of the energetic status. At the blood level it has been noted a decrease of $\beta$-hydroxybutyrate and non-esterified fatty acids. Beneficial effects have also been attributed to liver function, absorption of certain minerals and immune function.

In brief, the positive effect of monensin on the energy balance and the use of nutrients would reduce the mobilization of tissue reserves, playing down the risk of metabolic disorders, improving the health and therefore the performance of the dairy cow.

Key words: ionophores, ruminal metabolism, dairy cow.

\section{INTRODUCCIÓN}

El sector lácteo europeo y nacional, se encuentra actualmente en una difícil situación, consecuencia del desequilibrio del mercado y de la caída de los precios pagados a los productores. Desde el 1 de abril de 2015, con el final de la cuota láctea, la producción se encuentra liberalizada, no existiendo una restricción de la producción en los estados miembros de la Unión Europea que corrija el desequilibrio existente entre la oferta y la demanda de leche. Por tanto, el sector lácteo tiene que hacer frente a una gran volatilidad del precio de la leche, además del impacto de la variabilidad del precio de la energía y de las materias primas (MARM, 2016). Así, los productores necesitan abordar estrategias que mejoren su competitividad. En este sentido, la salud de las vacas es crucial para la rentabilidad de una explotación, ya que va a determinar su producción y su longevidad, y por tanto el periodo en el que el ganadero podrá amortizar su inversión.

\section{DESAFÍOS METABÓLICOS DURANTE EL PERIODO DE TRANSICIÓN}

Uno de los momentos más críticos en el ciclo productivo de la vaca lechera es el periodo de transición, definido como el periodo entre las tres semanas previas al parto y las tres semanas posteriores (Grummer, 1995). En el periodo de transición se combinan condiciones que comprometen la salud de la vaca, como son: una disminución de la ingesta de alimento que coincide con un aumento de los requerimientos de nutrientes (Ingvartsen \& Andersen, 2000); un aumento en la demanda de calcio con el inicio de la lactación (Horst, 1986); el estrés asociado al parto y cambios del lote; cambios endocrinos asociados al inicio del parto, lactogénesis y reanudación de la ciclicidad ovárica; y cambios en la dieta y en el comportamiento asociado al consumo de alimento (Kessel, Strohel, Meyer, Hiss, Sauerwein, Schwarz \& Bruckmeyer, 2008).

La importancia de este periodo se ha reconocido en numerosos artículos, ya que es donde 
se presenta una mayor incidencia de enfermedades (mastitis, cetosis, trastornos digestivos, cojeras, etc.) (Ingvartsen, Dewhurst \& Friggens, 2003). Además, los problemas de salud de la vaca normalmente no son eventos aislados, sino que un trastorno primario de salud suele desencadenar una secuencia de trastornos en cascada. Por ejemplo, las vacas con excesiva condición corporal en el periodo seco tienen más probabilidad de desarrollar hígado graso (Bobe, Young $\&$ Beitz, 2004) o de sufrir hipocalcemia (Houe, Ostergaard, Thilsing-Hansen, Jorgensen, Larsen, Sorensen, Agger \& Bloom, 2001; Ostergaard, Sorensen \& Houe, 2003). A su vez, la hipocalcemia exacerba la inmunosupresión (Ducusin, Uzuka, Satoh, Otani, Nishimura, Tanabe \& Sarashina, 2003; Kimura, Reinhardt \& Goff, 2006), y puede afectar a la eficiencia reproductiva, ya que las vacas que experimentan hipocalcemia presentan un mayor riesgo de enfermedad uterina y un retraso en la primera ovulación posparto (Witheford \& Sheldon, 2005).

Por tanto, el éxito con el que la vaca atraviese el periodo de transición va a condicionar su productividad durante la lactación. Sin embargo, dicha productividad no solo se debe al descenso de la producción sino también a la eliminación de animales a causa de la presentación de enfermedades durante este periodo tan crítico. Por otro lado, para lograr el objetivo económico de preñez dentro de los 120 días tras el parto, se debe restablecer un ciclo estral regular, el cual es el resultado de un complejo de interacciones y de señalizaciones endocrinas entre cerebro, hígado, ovarios y útero (Wathes, Fenwick, Cheng, Bourne, LLewellyn, Morris, Kenny, Murphy \& Fitzpatrick, 2007). Por ello, los trastornos que tienen lugar en el periodo de transición tienen consecuencias sobre la rentabilidad del productor mucho después de que esta fase finalice.

Durante la lactación temprana, la glándula mamaria exige un requerimiento de nutrientes superior al que es capaz de obtener a través de la ingestión de alimento, originando una si- tuación de balance energético negativo. Como consecuencia, la deficiencia de nutrientes se satisface mediante la movilización de reservas corporales (lípidos, proteínas, glucógeno y minerales) (Sovani, Heuer, Van Straalen \& Noordhuizen, 2000). Actualmente, existe una fuerte selección genética en las vacas de leche a favor de su capacidad para aumentar su producción convirtiéndolas en verdaderas "atletas metabólicas", como las denominan Chalupa y Harrison (1996).

Según la curva de producción de leche estándar en la vaca (Figura 1), tras el parto, se espera que la producción aumente hasta alcanzar el pico de máxima producción, momento a partir del cual comienza a descender. Por ello, la condición corporal (CC) va disminuyendo durante los primeros 2-3 meses, hasta el punto en que la vaca empieza a consumir suficiente para la producción y la CC comienza a recuperarse (Coffey, Simm, Hill \& Brotherstone, 2003).

La repercusión del balance energético negativo puede verse reflejado en las concentraciones circulantes de ácidos grasos no esterificados (NEFA) y $\beta$-hidroxibutirato (BHB) (LeBlanc, 2010). La concentración de NEFA refleja la magnitud de movilización de grasa desde los depósitos corporales y el nivel de ingesta de alimento (Adewuyi, Gruys \& Van Eerdenburg, 2005). Los cuerpos cetónicos (BHB, acetona y acetoacetato) son los metabolitos intermediarios de la oxidación de ácidos grasos a acetil CoA; así, el BHB refleja en qué grado se completa la oxidación de grasa en el hígado (LeBlanc, 2010). Conforme el aporte de NEFA al hígado supera la capacidad del hígado para oxidar completamente los ácidos grasos para aportar energía, la producción de cuerpos cetónicos aumenta (LeBlanc, 2010).

La dificultad en la adaptación al balance energético negativo puede predisponer a una variedad de enfermedades (Herdt, 2000), a una reducción de la eficiencia reproductiva (Buckley, O’Sullivan, Mee, Evans \& Dillon, 2003; Leroy, Vanholder, Mateusen, Christopher, Op- 


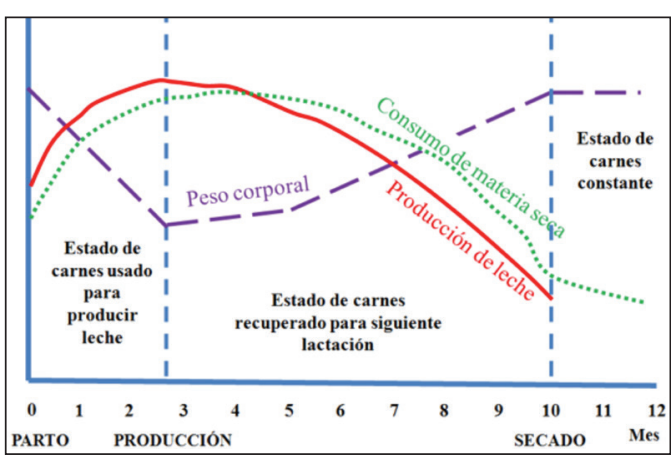

Figura 1. Variación en el consumo de materia seca, producción de leche y peso vivo durante la lactación de una vaca. (Adaptado de Chamberlain y Wilkinson, 2002)

somer, De Kruif, Genicot \& Van Soom, 2005; Leblanc, 2008), y a una exacerbación de la inmunosupresión en el periparto (Van Knegsel, De Vriesreilnigh, Meulenberg, Van Den Brand, Dijkstra, Kemp \& Parmentier, 2007). Así, según LeBlanc (2010), la cetosis subclínica (considerada cuando la concentración sérica de $\mathrm{BHB}$ es mayor a $1200 \mu \mathrm{mol} / \mathrm{l}$ ) en la primera o segunda semana después del parto está asociada con: un incremento de 3 a 8 veces el riesgo de desplazamiento de abomaso a la izquierda (Oetzel, 2004); un riesgo 3 veces mayor de metritis y de 4 a 6 veces mayor de cetosis clínica (Duffield, Lissemore, Mcbride \& Leslie, 2009); una mayor probabilidad de endometritis subclínica (Hammon, Evjen, Dhiman, Goff \& Walters, 2006); y una duración y severidad incrementadas de mastitis (Suriyasathaporn, Heuer, Noordhuizen-Stassen \& Schukken, 2000).

Por otro lado, en cuanto al efecto de la cetosis subclínica sobre la eficiencia reproductiva, Walsh, Kelton, Duffield, Leslie, Walston y Leblanc (2006) observaban que vacas con niveles de BHB en leche mayores a $1000 \mu \mathrm{mol} / \mathrm{l}$ en la primera semana posparto tenían 1,5 veces más probabilidades de ser anovulatorias a las 9 semanas posparto. Estos mismos investigadores posteriormente, mostraban como vacas con cetosis en la primera o segunda semana posparto aumentaban el intervalo medio hasta quedar gestantes, e incluso podía alargarse más (hasta 130 días) en vacas que tuvieron cetosis subclínica tanto en la primera como en la segunda semana de lactación (Walsh, Leslie, Leblanc, Kelton, Walton \& Duffield, 2007).

\section{UTILIZACIÓN DE MONENSINA PARA MEJORAR LA EFICIENCIA PRODUCTI- VA EN VACAS DE LECHE}

Una manera de ayudar a aliviar el balance energético negativo de la vaca en el periodo de transición es mediante el uso de ionóforos, que actúan modificando la población microbiana del rumen (Bergen \& Bates, 1984). Los ionóforos como la lasalocida y la monensina (poliéteres carboxílicos) se han usado como aditivo alimentario en vacuno desde la década de 1970 para mejorar la eficiencia alimentaria (Jerez, Chihuailaf, Gai, Noro \& Wittwer, 2014), entre ellos, la monensina (MON) producida por una cepa natural de Streptomyces cinnamonensis es el más comúnmente utilizado (Duffield, Rabiee \& Lean, 2008). Sin embargo, con la entrada en vigor del Reglamento 1831/2003 de 22 de septiembre de 2003 sobre los aditivos en la alimentación animal, su uso como aditivo promotor del crecimiento en pienso quedó prohibido partir del 1 de enero de 2006. Actualmente la MON es catalogada como sustancia antiinfecciosos/antibióticos y su uso está aprobado por la Comisión Europea para su utilización en vacas (Reglamento 37/2010 relativo a las sustancias farmacológicamente activas y su clasificación por lo que se refiere a los límites máximos de residuos en los productos alimenticios de origen animal). Debido a sus propiedades físico-químicas, la MON no se transfiere a la leche, por lo que a las dosis recomendadas de utilización, no es detectada en la misma (Jerez et al., 2014).

La MON inhibe selectivamente el crecimiento de bacterias mediante la alteración de la transferencia de iones a través de la membrana 
celular, siendo las bacterias Gram positivas más sensibles que las Gram negativas. De esta forma provoca una disminución de la microflora productora de acetato y butirato, y un incremento de la microflora que produce propionato, un percursor gluconeogénico, resultando en un incremento de la gluconeogénesis hepática. Así, el mayor suministro de glucosa se asume como el principal beneficio de la suplementación con MON (Mullins, Mamedova, Brouk, Moore, Green, Perfield, Smith, Harner \& Bradford., 2012). De hecho, en vacas tratadas con MON se ha observado un aumento de la eficiencia del propionato para producir glucosa y un disminución de la oxidación de glucosa en los tejidos corporales (Markantonatos \& Varga, 2017). Este efecto es importante en las vacas de leche en el periodo de transición, ya que experimentan un incremento en la demanda de glucosa y un descenso de su disponibilidad (Petersson-Wolfe, Leslie, Osborne, Mcbride, Bagg \& Vessie, 2007). Por otro lado, observaciones de algunos estudios han sugerido que los efectos beneficiosos de la MON pueden ser independiente a los cambios en la gluconeogénesis (Mullins et al., 2012). Así, Markantonatos, Aharoni, Richardson \& Varga (2009) observaron que la MON no afectaba la producción de propionato en el rumen al medir su cinética durante el periodo del periparto.

Otros efectos atribuidos a la MON han sido la menor digestión de la proteína en el rumen con el consiguiente incremento de la disponibilidad de la proteína alimentaría en el intestino delgado (McGuffey, Richardson \& Wilkinson, 2001), aunque se ha sugerido que estos efectos pueden verse más o menos limitados en función del tipo de dieta (Tedeschi, Fox \& Tylutki, 2003). También se ha observado una influencia de la MON sobre las concentraciones séricas de urea, produciendo un aumento en sus niveles séricos (Duffield, Sandals, Leslie, Lissemore, Mcbride Lumsden, Dick \& Bagg, 1998) y se sugiere que está relacionado con su potencial de incrementar la absorción de aminoácidos en el intestino para su metabolismo hepático
(Plaizier, Martin, Duffield, Bagg, Dick \& Mcbride, 2000), aunque otra hipótesis es que la MON podría tener efectos beneficiosos sobre la funcionalidad hepática y por tanto mejorar la habilidad del hígado para la producción de urea (Petersson-Wolfe et al., 2007).

Otras investigaciones sobre la vaca de transición indican un efecto beneficioso de la MON en la curva de ingesta de materia seca (IMS) posparto (Shah, Schroeder, Strang \& Green, 2008), lo que también contribuiría a mejorar la salud, ya que el balance energético negativo en las vacas en lactación temprana con IMS más elevadas no es tan severo como en las vacas con IMS inferiores (Bertics, Grumme, Cadorniga-Valino \& Stoddard, 1992). Sin embargo, Duffield et al. (2008) tras la realización de un meta análisis sobre los efectos de la MON concluyen que el tratamiento de MON en vacas lecheras disminuye la ingestión de materia seca, y Peterson-Wolfe, Leslie, Osborne, McBride, Bagg, Vessie, Dick \& Duffieldet (2007) no encontraron diferencias en la ingestión de materia seca entre vacas tratadas con MON y sin tratar, por lo que el efecto de la MON sobre la ingestión no es muy claro.

Según Spears, Schricker y Burns (1989), el tratamiento con MON también puede incrementar la absorción de Ca desde el intestino, así como la absorción de Se (Costa, Gleed, Sanson, Symonds \& Allen, 1985) y Cu (Van Ryssen, 1991); estos minerales tienen un papel importante en los mecanismos de acción antioxidantes. Deficiencias en selenio se han asociado con un incremento en la incidencia de infección uterina (Harrison, Hancock, Pierre, Conrad \& Harvey, 1986), por lo que una mayor disponibilidad de estos minerales podría modificar el riesgo de enfermedad en el periparto.

Diferentes estudios han indicado efectos beneficiosos del tratamiento con MON sobre metabolitos sanguíneos, y parámetros productivos y reproductivos de la vaca en periodo de transición, aunque los resultados entre estudios son variables, dependiendo, entre otros factores, de 
la forma o el momento de administración. Por ejemplo, la MON puede aportarse en forma de premezcla, que se añade directamente al alimento, o en forma de una cápsula de liberación continua (CLC) como dispositivo intrarruminal que se administra 3-4 semanas antes del parto. Se ha especulado que la forma de premezcla no pueda proporcionar los mismos beneficios que la CLC, debido a la fluctuación en la cantidad de principio activo en el rumen al disminuir el consumo de alimento en torno al parto (Petersson-Wolfe et al., 2007). El dispositivo intrarruminal libera lentamente el principio activo en el rumen durante aproximadamente 100 días (McGuffey et al., 2001), influyendo en el metabolismo ruminal de la vaca durante la fase de preparto y posparto.

En el caso de la MON suministrada en forma de CLC, los estudios que se han llevado a cabo para evaluar sus efectos sobre metabolitos sanguíneos, concluyen principalmente que produce una reducción en las concentraciones séricas de BHB y NEFA (Duffield, Leblanc, Bagg, Leslie, Ten Hag \& Dick., 2003a; Peterson-Wolfe et al., 2007; Mullins et al., 2012), mientras que los resultados acerca de otros metabolitos como glucosa, urea, y bilirrubina no son tan consistentes (Duffield et al., 2008). También existen diferentes estudios que indican que el uso de MON en forma de premezcla reduce las concentraciones séricas de BHB y NEFA (Thomas, Poe, Mcguffey, Mowrey \& Allrich, 1993; Heuer, Schukken, Jonker, Wilkinson \& Noordhuizen, 2001; Vallimont, Varga, Arieli, Cassidy \& Cummins, 2001) y mejora la disponibilidad de glucosa (Arieli, Vallimont, Aharoni \& Varga, 2001).

El efecto positivo de la MON sobre el balance energético y la utilización de nutrientes reduciría la movilización de reservas tisulares, minimizando el riesgo de desórdenes metabólicos, mejorando la salud y por tanto el rendimiento de la vaca. Por ello, diferentes autores han indicado que el tratamiento con MON tiene el potencial de reducir el riesgo de enfermedad posparto y aumentar la producción de leche (Duffield et al., 2008; Arieli, Dicken, Dagoni, Spirer \& Zamwel, 2008). Si bien, en otros estudios se han encontrado una asociación negativa entre la utilización de MON y algunos componentes de la leche como el porcentaje de grasa (Duffield, Bagg, Kelton, Dick \& Wilson, 2003b).

Algunos ensayos atribuyen un efecto beneficioso de la MON sobre el sistema inmune, al encontrar mayor tasa de anticuerpos específicos de la diarrea vírica bovina en vacas vacunadas y tratadas con MON frente a las no tratadas (Drong, Meyer, von Soosten, Frahm, Rehage, Schirrmeier, Beer \& Danicke, 2016). Recientemente, en estudios realizados in vitro sobre la fermentación ruminal, Capelari, Johnson, Latack, Roth \& Powers (2018) observaban una disminución de la producción de metano al incorporar MON a la dieta de vacas lecheras.

A pesar de los posibles beneficios del uso de MON, existen otros estudios en los que no se han encontrado estos efectos beneficiosos del tratamiento con MON, sobre parámetros productivos o reproductivos (Salar, Çalisici, Çalisici, Bastan \& Bastan, 2017), ni sobre excreción y utilización de nitrógeno, fósforo ni azufre (Morris, Kim, \& Lee, 2018). Esta discordancia entre resultados puede atribuirse a la heterogeneidad de las condiciones en las que se llevan a cabo los estudios y a la influencia de numerosos factores, como la dieta, el manejo, o la genética, entre otros.

En conclusión, aunque el tratamiento con MON parece ser beneficioso para el sector vacuno lechero, la variación de resultados entre algunos de estudios realizados, hace necesario ampliar el número de ensayos y el tamaño de la población estudiada para poder evaluar eficazmente la influencia de tratamientos con MON autorizadas que puedan resultar de gran interés para los productores, ya que un exitoso periodo de transición es fundamental para un adecuado desarrollo del potencial productivo de la vaca de leche y por tanto condiciona en gran medida su rentabilidad. 


\section{BIBLIOGRAFÍA}

Adewuyi, A., Gruys, E., y Van Eerdenburg, F.C.M. (2005). Non esterified fatty acids (NEFA) in dairy cattle. A review. Vet Q. 27: 117-116.

Arieli, A., Vallimont, J.E., Aharoni, Y., y Varga, G.A., (2001). Monensin and growth hormone effects on glucose metabolism in the prepartum cow. J. Dairy Sci. 84:2770-2776.

Arieli, A., Dicken, U., Dagon, I., Spirer, Y., y Zamwel, S., (2008). Production and Health of Cows Given Monensin Prepartum and a High-Energy Diet Postpartum. J. Dairy Sci. 91:1845-1851.

Bergen, W., y Bates, D.B. (1984). Ionophores: Their effect on production efficiency and mode of action. J. Anim. Sci. 58:1528-1539

Bertics, S.J., Grumme, R.R., CadornigA-Valino, C., y Stoddard, E.E., (1992). Effect of prepartum dry matter intake on liver triglyceride concentration and early lactation. J. Dairy Sci. 75:1914-1922.

Bobe, G., Young, J.W., y Beitz, D.C. (2004). Pathology, etiology, prevention and treatment of fatty liver in dairy cows. J. Dairy Sci. 87:3105-3124.

Buckley, F., O’Sullivan, K., Mee, J.F., Evans, R.D., y Dillon, P. (2003). Relationships among milk yield, body condition, cow weight and reproduction in Spring-Calved Holstein-Friesians. J. Dairy Sci. 86:23082319.

Capelari, M., Johnson, K.A., Latack, B., Roth, J., y Powers, W. (2018). The effect of encapsulated nitrate and monensin on ruminal fermentation using a semi-continuous culture system. J. Anim. Sci. 96 (8):34463459.

Chalupa, W.Y., y Harrison, J.H. (1996). Feeding Strategies for the Fresh Cow. The Penn Annual Conference Center for Animal Health and Productivity. University of Pennsylvania. Consultado on line el 15/02/2017 en: http://research.vet.upenn.edu/DairyPoul-
trySwine/DairyCattle/PennConf1996/FeedingStrategiesfortheFreshCow/tabid/1728/ Default.aspx

Chamberlain, A.T., y Wilkinson, J.M., (2002). Feeding and dairy cows. Edición en lengua española. Editorial Acribia. 318 pp.

Coffey, M.P., Simm, G., Hill, W.G., y Brotherstone, S., (2003). Genetic Evaluations of Dairy Bulls for Daughter Energy Balance Profiles Using Linear Type Scores and Body Condition Score Analyzed Using Random Regression. J. Dairy Sci. 86 (6): 2205-12.

Costa, N.D., Gleed, P.T., Sanson, B.F., Symonds, H.W., y Allen W.M. (1985). Monensin and narasin increase selenium and zinc absorption in steers. Page 472 in Trace Element Metabolism in Man and Animals. C. F. Mills, I. Bremner, and J. K. Chesters, ed. Commonw. Agric. Bur., Slough, England.

Drong,C., Meyer, U., von Soosten, D., Frahm, J., Rehage, J., Schirrmeier, H., Beer, M., y S. Danicke (2016). Effects of monensin and essential oils on immunological, haematological and biochemical parameters of cows during the transition period. J. Anim. Physiol. Anim. Nutr. 100 (3):537-51.

Ducusin, R.J., Uzuka, Y., Satoh, E., Otani, M., Nishimura, M., Tanabe, S., y Sarashina, T. (2003). Effects of extracelular $\mathrm{Ca}^{2+}$ on phagocytosis and intracelular $\mathrm{Ca}^{2+}$ concentrations in polymorphonuclear leukocytes of postpartum dairy cows. Res.Vet. Sci. 75:27-32.

Duffield, T.F., Sandals, D., Leslie, K.E., Lissemore, K., Mcbride Lumsden, B.W., Dick, J.H., y Bagg, R. (1998). Efficacy of monensin for the prevention of subclinical ketosis in lactating dairy cows. J. J. Dairy Sci. 81:2866-2873.

Duffield, T.F., Leblanc, S., Bagg, R., Leslie, K., Ten Hag, J., y Dick, P. (2003a). Effect of a Monensin Controlled Release Capsule on Metabolic Parameters in Transition Dairy Cows. J. Dairy Sci. 86:1171-1176.

Duffield, T.F., Bagg, R., Kelton, D., Dick, P y Wilson, J. (2003b). A Field Study of Di- 
etary Interactions with Monensin on Milk Fat Percentage in Lactating Dairy Cattle. J. Dairy Sci. 86:4161-4166.

Duffield, T.F., Rabiee, A.R., y Lean, I.J. (2008). A meta-analysis of the impact of monensin in lactating dairy cattle. Part 1. Metabolic effects J. Dairy Sci. 91:1334-1346.

Duffield, T.F., Lissemore, K.D., Mcbride, B.W., y Leslie, K.E. (2009). Impact of hyperketonemia in early lactation dairy cows on health and production. J. Dairy Sci. 92: 571-580.

Grummer, R.R. (1995). Impact of changes in organic nutrient metabolism on feeding the transition cow. J. Anim. Sci. 73:2820-2833.

Hammon, D.S., Evjen, I.M., Dhiman, T.R., Goff, J.P., y Walters, J.L. (2006). Neutrophil function and energy status in Holstein cows with uterine health disorders. Vet. Immunol. Immunopathol. 113:21-29.

Harrison, J.H., Hancock, D.D., Pierre, N., Conrad, H.R., y Harvey, W.R. (1986). Effect of prepartum selenium treatment on uterine involution in the dairy cow. J. Dairy Sci. 69:1421.

Herdt, T.H. (2000). Ruminant adaptation to negative energy balance. Vet. Clin. North Am. Food Anim. Pract. 16: 215-230.

Heuer, C., Schukken, Y.H., Jonker, L.J, Wilkinson, J.I., y Noordhuizen, J.P. (2001). Effect of monensin on blood ketone bodies, incidence and recurrence of disease and fertility in dairy cows. J. Dairy Sci. 84:1085-1097.

Horst, R.L. (1986). Regulation of calcium and phosphorus homeostasis in the dairy cows. J. Dairy Sci. 69:604.

Houe, H., Ostergaard, S., Thilsing-Hansen, T., Jorgensen, R.J., Larsen, T., Sorensen, J.T., Agger, J. F., y Bloom, J.Y. (2001). Milk fever and subclinical hypocalcaemia - an evaluation of parameters on incidence risk, diagnosis, risk factors and biological effects as input for a decision support system for disease control. Acta Vet. Scand. 42:1-29.

Ingvartsen, K.L., y Andersen, J.B. (2000). Integration of metabolism and intake regula- tion: A review focusing on periparturient animals. J. Dairy Sci. 83:1573-1597.

Ingvartsen, K.L., Dewhurst, R.J., y Friggens, N.C. (2003). On the relationship between lactational performance and health: is it yield or metabolic imbalance that cause production diseases in dairy cattle? A position paper. Livest. Prod. Sci. 83:277-308.

Jerez, A., Chihuailaf, R., Gai, M., Noro, M., y Wittwer, F. (2014). Detection of lasalocid and monensin in raw milk samples from supplemented dairy cows. Arch. Med. Vet. 46: 445-449.

Kessel, S., Strohel, M., Meyer, H.H.D., Hiss, S., Sauerwein, H., Schwarz, F.J., y Bruckmeyer, R.M. (2008). Individual variability in physiological adaptation to metabolic stress during early lactation in dairy cows kept under equal conditions. J. Anim. Sci. T. 86. P. 2903-2912

Kimura, K., Reinhardt, T.A., y Goff, J.P. (2006). Parturition and hypocalcemia blunts calcium signals in immune cells of dairy cattle. J. Dairy Sci. 89:2588-2595.

Leblanc, S.J. (2008). Postpartum uterine disease and dairy herd reproductive performance: a review. Vet J. 176: 102-114.

Leblanc, S. (2010). Monitoring metabolic health of dairy cattle in the transition period. J. Reprod. Dev. 56(S), S29-S35.

Leroy, J.L., Vanholder, T., Mateusen, B., Christopher, A., Opsomer, G., De Kruif, A., Genicot, G., y. Van Soom, A. (2005). Non-esterified fatty acids in follicular fluid of dairy cows and their effect on developmental capacity of bovine oocytes in vitro. Reproduction 130: 485-495.

Markantonatos, X., Aharoni, Y., Richardson, L.F., y Varga, G.A. (2009). Effecs of monensin on volatile fatty acid metabolism in periparturient dairy cows using compartmental analysis. Anim. Feed Sci. Technol. 153:11-27.

Markantonatos, X., y Varga, G.A. (2017). Effects of monensin on glucose metabolism 
in transition dairy cows. J. Dairy Sci. 100:9020-9035.

MARM (Ministerio de agricultura, alimentación y medio ambiente), Gobierno de España. (2016). Accedido el 01/02/2017 en: http:// www.mapama.gob.es/es/ganaderia/temas/ produccion-y-mercados-ganaderos/sectoresganaderos/vacuno-lechero/default.aspx

Mcguffey, R.K., Richardson, L.F., y Wilkinson, J.I.D. (2001). Ionophores for dairy cattle: Current status and future outlook. J. Dairy Sci. 84:194-203.

Morris, D., L. Kim, S. H. y Lee, C. (2018). Effects of corn feeding reduced-fat distillers grains with or without monensin on nitrogen, phosphorus, and sulfur utilization and excretion in dairy cows. J. Dairy Sci. 101:7106-7116.

Mullins, C.R., Mamedova, L.K., Brouk, M.J., Moore, C.E., Green, H. B., Perfield, K.L., Smith J.F., Harner, J.P., y Bradford, B.J. (2012). Effects of monensin on metabolic parameters, feeding behavior, and productivity of transition dairy cows. J. Dairy Sci. 95:1323-1336.

Oetzel, G.R. (2004). Monitoring and testing dairy herds for metabolic disease. Vet. Clin N Amer. Food Anim. 20: 651-674.

Ostergaard, S., Sorensen, J.T., y Houe, H. (2003). A stochastic model simulating milk fever in a dairy herd. Prev. Vet. Med. 58:125-143.

Petersson-Wolfe, C.S., Leslie K.E., Osborne, T., Mcbride, B.W., Bagg R., y Vessie, G. (2007). Effect of monensin delivery method on dry matter intake, body condition score, and metabolic parameters in transition dairy cows. J. Dairy Sci. 90:1870-1879.

Plaizier, J.C., Martin, A., Duffield, T., Bagg, R., Dick, P., y Mcbride, B.W. (2000). Effect of a prepartum administration of monensin in a controlled-release capsule on apparent digestibilities and nitrogen utilization in transition dairy cows. J. Dairy Sci. 83:2918-2925.
REGLAMENTO (CE) No 1831/2003 DEL PARLAMENTO EUROPEO Y DEL CONSEJO de 22 de septiembre de 2003 sobre los aditivos en la alimentación animal. Diario Oficial de la Unión Europea. L 268/29.

REGLAMENTO (UE) No 37/2010 DE LA COMISIÓN de 22 de diciembre de 2009 relativo a las sustancias farmacológicamente activas y su clasificación por lo que se refiere a los límites máximos de residuos en los productos alimenticios de origen animal. Diario Oficial de la Unión Europea. L 15/1.

Salar, S., Çalisici, O., Çalisici, D., Bastan, I., y Bastan, A. (2017). Effect of utilization of a controlled-release intraruminal monensin device on dry matter intake, milk yield, and some reproductive parameters in dairy cows. Turk J Vet. Anim. Sci. 41: 621-626.

Shah, M.A., Schroeder, G., Strang, B.D., y Green, H.B. (2008.) Effect of monensin concentration on dry matter intake during the transition period of lactating dairy cows. J. Dairy Sci. 91 (E Suppl. 1): 268-269.

Sovani, S., Heuer, C., Van Straalen, W.M., y Noordhuizen, J.P.T.M. (2000). Disease in high producing dairy cowsfolloging post parturient negative energy balance. Pages 33-50 in Soc. Vet. Epid. Prev. Proc. Edinburgh, UK, March 29-31, 2000. Soc. Vet. Epidemiol. Prev. Med., UK.

Spears, J.W., Schricker, B.R.R., y Burns, J.C. (1989). Influence of lysocellin and monensin on mineral metabolism of steers fed forage-based diets. J. Anim. Sci. 67:2140.

Suriyasathaporn, W., Heuer, C., NoordhuizenStassen, E.N., y Schukken, Y.H. (2000). Hyperketonemia and udder defense: a review. Vet Res; 31: 397-412.

Tedeschi, L.O., Fox, D.G., y Tylutki, T.P. (2003). Potential environmental benefits of ionophores in ruminant diets. J. Environ. Qual. 32:1591-1602.

Thomas, E.E., Poe, S.E., Mcguffey, R.K., Mowrey, D.H., y Allrich, R.D. (1993). Effect of feeding monensin to dairy cows on 
milk production and serum metabolites during early lactation. J. Dairy Sci. 76 (Suppl. 1):280. (Abstr.).

Vallimont, J.E., Varga, G.A., Arieli, A., Cassidy, T.W., y Cummins, K.A. (2001). Effects of prepartumsomatotropin and monensin on metabolism and production of periparturient Holstein dairy cows. J. Dairy Sci. 84:2607-2621.

Van KnegseL, A.T.M., DE Vriesreilnigh, G., Meulenberg. S., Van Den Brand, H., Dijkstra, J., Kemp B., y Parmentier, H.K. (2007). Natural antibodies related to energy balance in early lactation dairy cows. J. Dairy Sci. 90:5490-5498.

Van Ryssen, J.B.J. (1991). Effect of monensin and its metabolites in broiler litter on sheep consuming the broiler litter. J. S. Afr. Vet. Med. Assoc. 62:94.
Walsh, R.B., Kelton, D.F., Duffield, T., Leslie K.E., Walston J.S., y Leblanc S.J. (2006). Prevalence and risk factors for postpartum anovulatory condition in dairy cows. J. Dairy Sci. 90:315-324.

Walsh, R., Leslie, K., Leblanc, S., Kelton, D., Walton, J., y Duffield, T. (2007). The effect of subclinical ketosis in early lactation on reproductive performance of postpartum dairy cows. J. Dairy Sci; 90: 2788-2796.

Wathes, D.C., Fenwick, M., Cheng, Z., Bourne, N., LLewellyn, S., Morris, D.G, Kenny, D., Murphy, J., y Fitzpatrick, R. (2007). Influence of negative energy balance on cyclicity and fertility in the high producing dairy cow. Theriogenology, 68: S232-S241.

Whiteford, L.C., y Sheldon, I.M. (2005). Association between clinical hypocalcaemia and postpartum endometritis. Vet. Rec. 157:202204. 